\title{
Field evaluation of the entomopathogenic fungi enriched with organic amendments against Holotrichia consanguinea Blanchard (Coleoptera: Scarabaeidae) infesting groundnut
} crop

\author{
Prakash S. Patel, Sushma Deb, Prakash H. Rabari and Manishkumar J. Joshi* (0)
}

\begin{abstract}
Background: White grubs are highly polyphagous and most destructive soil pests inflicting damage to a groundnut crop that causes economic yield losses in commercial groundnut production in India. In this study, potential of the two entomopathogenic fungi (EPF) viz., Metarhizium anisopliae (Metchnikoff) Sorokin and Beauveria bassiana (Balsamo) Vuillemin enriched with organic amendments like neem cake, castor cake, vermicompost and poultry manure was evaluated to manage buildup of white grub population in groundnut crop.

Results: Results showed that the plot treated with soil application of vermicompost @ 1 ton/ha + M. anisopliae 1.15 WP $\left(1 \times 10^{8} \mathrm{cfu} / \mathrm{g}\right) @ 2 \mathrm{~kg} / \mathrm{ha}$ showed the least amount of plant mortality due to white grub which was at par with

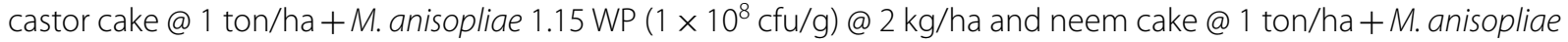
1.15 WP $\left(1 \times 10^{8} \mathrm{cfu} / \mathrm{g}\right) @ 2 \mathrm{~kg} / \mathrm{ha}$ which showed only 1.74, 2.00 and $2.24 \%$ plant damage, respectively. Plots treated with vermicompost @ 1 ton/ha + M. anisopliae 1.15 WP $\left(1 \times 10^{8} \mathrm{cfu} / \mathrm{g}\right) @ 2 \mathrm{~kg} / \mathrm{ha}$ had the fewest (0.19) white grubs per one meter row length. Based on highest pod yield, net realization and net gain, the treatment with soil application of vermicompost @ 1 ton/ha + M. anisopliae 1.15 WP $\left(1 \times 10^{8} \mathrm{cfu} / \mathrm{g}\right) @ 2 \mathrm{~kg} / \mathrm{ha}$ was found most effective treatments for the management of white grub in groundnut.
\end{abstract}

Conclusions: Results indicated that M. anisopliae supplemented with various organic amendments, such as vermicompost, castor cake, and neem cake can be commercialized as a healthy alternative to minimize the use of synthetic pesticides in groundnut crops for white grub management.

Keywords: Entomopathogenic fungi, Metarhizium anisopliae, Beauveria bassiana, Vermicompost, Neem cake, White grub, Soil application

\section{Background}

Groundnut or peanut (Arachis hypogaea L.) is known as "King of oilseed crops" and considered one of the most important oil seed crop in India, as well as it occupied

\footnotetext{
*Correspondence: mj1107006@gmail.com

Department of Entomology, S. D. Agricultural University,

Sardarkrushinagar, Gujarat, India
}

the fourth rank among important edible oil sources in the world. It originated in South America and contains $48-50 \%$ oil and $26-28 \%$ protein (Janila et al. 2013). In India, Gujarat is the largest producer contributing $25 \%$ of the total production, followed by Tamil Nadu (22.48\%). It is valued as the fourth most important source of edible oil and the third most important source of vegetable protein (Deepthi 2014). The crop can be grown successfully 
in areas receiving rainfall from 600 to $1250 \mathrm{~mm}$. Groundnut crop prefers sandy loam, loamy and medium black. The crop is grown as monoculture in Banas kantha region of Gujarat.

The plant suffers from the ravages of insect-pests and a loss in production both in terms of quality and quantity. As many as 52 species of insect-pests and 2 species of mites have been recorded infesting the groundnut crop in India (Singh et al. 1990). In India, the grubs of Holotrichia consanguinea Blanch (Coleoptera: Scarabaeidae) are of major economic importance attacking groundnut in Gujarat, Rajasthan, Bihar, Uttar Pradesh, Haryana and Punjab (Chandel et al. 2015). White grubs are polyphagous and they can feed on any root or underground stem (Veeresh 1988). The first instar larvae feed in part on organic matter in the soil, while the second and third instar grubs feed largely on roots or underground stems. The economic importance of chafers is primarily due to the feeding activity of the third instar grubs (Chandel et al. 2015).

The current method for the control of white grubs is through the use of chemical pesticides. However, concern about safety, environmental contamination and poor efficacy of recommended insecticides has increased the need to develop integrated pest management (IPM) approaches for these pests (Kumawat 2001). The emphasis has shifted from the dominant chemical pesticides to IPM, where the focus is on biological control and other natural resources with reduced reliance on chemicals. Yadava and Sharma (1995) have reported that several EPF such as Metarhizium anisopliae (Metchnikoff) Sorokin, Beauveria bassiana (Balsamo) Vuillemin, B. brongniartii (Saccardo) are pathogenic to white grubs and are effective in suppressing their population under field conditions.
Therefore, the present study aimed to evaluate the potential of EPF enriched with organic amendments against the white grub, $H$. consanguinea under field conditions.

\section{Methods \\ Experimental site}

Field trials were conducted at Agronomy Instructional Farm, C. P. College of Agriculture, SDAU, Sardarkrushinagar. This site is situated at longitude $24.3217^{\circ} \mathrm{N}$, latitude $72.3177^{\circ} \mathrm{E}$ and with elevation $172 \mathrm{~m} / 564 \mathrm{ft}$ ASL. Area represents the typical climatic conditions of semi-arid regions having low rainfall with a wide chasm between diurnal and nocturnal temperatures. The soils are deep loamy sand to sandy loam. The general topography of the swathe is rugged plain.

\section{Field evaluation}

Groundnut was raised in Kharif for 3 consecutive seasons viz., 2018, 2019 and 2020 by following standard agronomical practices. The local variety GG-2 was sown at spacing of $45 \mathrm{~cm} \times 10 \mathrm{~cm}$ with a seed rate of $100 \mathrm{~kg} / \mathrm{ha}$. The experimental methodology was randomized block design (RBD), which split the entire farmed area into 13 equal plots $(4.00 \mathrm{~m} \times 3.15 \mathrm{~m})$. Each treatment was repeated 3 times, and each plot was individually treated with one of the tested 12 treatments at a specified concentration. As a control, one plot was left untreated. The plot for each treatment followed all the recommended package of practices except white grub management. The calculated amount EPF viz., M. anisopliae and $B$. bassiana was mixed with different organic amendments 20 days prior to land preparation (Table 1 ). The treatments were applied at the time of land preparation. Observation on germination was recorded after 15 days

Table 1 Treatment details

\begin{tabular}{|c|c|}
\hline Sr. no & Treatments \\
\hline 1 & Neem cake@ 1 ton/ha \\
\hline 2 & Neem cake@ 1 ton/ha + Beauveria bassiana 1.15WP $\left(1 \times 10^{8} \mathrm{cfu} / \mathrm{g}\right) @ 2$ kg/ha \\
\hline 3 & Neem cake@ 1 ton/ha + Metarhizium anisopliae 1.15 WP $\left(1 \times 10^{8} \mathrm{cfu} / \mathrm{g}\right) @ 2$ kg/ha \\
\hline 4 & Poultry manure @ 1 ton/ha \\
\hline 5 & Poultry manure@ @ ton/ha + Beauveria bassiana 1.15WP $\left(1 \times 10^{8} \mathrm{cfu} / \mathrm{g}\right) @ 2$ kg/ha \\
\hline 6 & Poultry manure@1 ton/ha + Metarhizium anisopliae 1.15 WP $\left(1 \times 10^{8} \mathrm{cfu} / \mathrm{g}\right) @ 2 \mathrm{~kg} / \mathrm{ha}$ \\
\hline 7 & Vermicompost @ 1 ton/ha \\
\hline 8 & Vermicompost@1ton/ha +Beauveria bassiana 1.15WP $\left(1 \times 10^{8} \mathrm{cfu} / \mathrm{g}\right) @ 2$ kg/ha \\
\hline 9 & Vermicompost@1 ton/ha + Metarhizium anisopliae 1.15WP $\left(1 \times 10^{8} \mathrm{cfu} / \mathrm{g}\right) @ 2$ kg/ha \\
\hline 10 & Castor cake @ 1 ton/ha \\
\hline 11 & Castor cake@ 1 ton/ha + Beauveria bassiana 1.15WP $\left(1 \times 10^{8} \mathrm{cfu} / \mathrm{g}\right) @ 2$ kg/ha \\
\hline 12 & Castor cake@1 ton/ha+Metarhizium anisopliae 1.15WP $\left(1 \times 10^{8} \mathrm{cfu} / \mathrm{g}\right) @ 2$ kg/ha \\
\hline 13 & Untreated control \\
\hline
\end{tabular}


of sowing from each net plot by recording total number of plants. Total number of plants and plants damaged by white grub were recorded at 20,40, 60 and 80 days after germination (DAG) from net plot. The damaged plants were removed after each count. From these data, percent plant mortality due to white grub was computed. After harvesting, white grub population was recorded from 3 rows of one-meter area in each plot by digging soil. Yield was also recorded at harvest and economics of treatments was calculated.

\section{Statistical analysis}

Observed data of plant mortality and number of white grub/1 $\mathrm{m}$ row were analyzed on the pattern of a factorial randomized block design (FRBD). All obtained data were statistically subjected to analysis of variance (ANOVA) through the SPSS Computer program (IBM SPSS
Statistics for Windows, Version 20.0. Armonk, NY: IBM Corp.). Significance of differences between the treatment means was compared by Duncan's multiple range tests (DNMRT) at $P \leq 0.05$ (Gomez and Gomez 1984).

\section{Results}

Effect of entomopathogenic fungi enriched with organic amendments on plant mortality (\%)

Results showed that there was non-significant difference on plant mortality (\%) due to white grub infestation in all the treatments, 20 days after germination during seasons of 2018, 2019 and 2020 (Tables 2, 3 and 4). In the year 2018 (Table 2), at 40 days after germination the minimum plant mortality was observed in the treatment with vermicompost @ 1 ton/ha $+M$. anisopliae 1.15 WP $\left(1 \times 10^{8} \mathrm{cfu} / \mathrm{g}\right) @ 2 \mathrm{~kg} / \mathrm{ha}$ and it was at part with castor cake @ 1 ton/ha + M. anisopliae

Table 2 Effect of entomopathogenic fungi enriched with organic amendments against white grub in groundnut (season of 2018)

\begin{tabular}{|c|c|c|c|c|c|c|}
\hline \multirow[t]{2}{*}{ Tr. no } & \multirow[t]{2}{*}{ Treatments } & \multicolumn{5}{|c|}{ Dead plants (\%) } \\
\hline & & $1-20$ DAG & $21-40$ DAG & 41-60 DAG & $61-80$ DAG & Mean over period \\
\hline 1 & Neem cake@1 ton/ha & $\begin{array}{l}5.25^{\mathrm{ab}} \\
(0.84)\end{array}$ & $\begin{array}{l}9.25^{\text {de }} \\
(2.60)\end{array}$ & $\begin{array}{l}11.01^{f} \\
(3.65)\end{array}$ & $\begin{array}{l}13.56^{\mathrm{e}} \\
(5.55)\end{array}$ & $\begin{array}{l}9.77^{\mathrm{fg}} \\
(2.88)\end{array}$ \\
\hline 2 & $\begin{array}{l}\text { Neem cake @ } 1 \text { ton/ha + Beauveria bassiana 1.15 WP } \\
\left(1 \times 10^{8} \mathrm{cfu} / \mathrm{g}\right) @ 2 \mathrm{~kg} / \mathrm{ha}\end{array}$ & $\begin{array}{l}4.69^{b c} \\
(0.67)\end{array}$ & $\begin{array}{l}8.77^{\mathrm{ef}} \\
(2.34)\end{array}$ & $\begin{array}{l}10.43^{\mathrm{fg}} \\
(3.33)\end{array}$ & $\begin{array}{l}12.81^{\mathrm{e}} \\
(4.97)\end{array}$ & $\begin{array}{l}9.17^{9} \\
(2.54)\end{array}$ \\
\hline 3 & $\begin{array}{l}\text { Neem cake @ } 1 \text { ton/ha + Metarhizium anisopliae 1.15 WP } \\
\left(1 \times 10^{8} \mathrm{cfu} / \mathrm{g}\right) @ 2 \mathrm{~kg} / \mathrm{ha}\end{array}$ & $\begin{array}{l}5.03^{\mathrm{abc}} \\
(0.77)\end{array}$ & $\begin{array}{l}6.74^{f} \\
(1.41)\end{array}$ & $\begin{array}{l}8.62^{g h} \\
(2.25)\end{array}$ & $\begin{array}{l}10.25^{f} \\
(3.40)\end{array}$ & $\begin{array}{l}7.66^{h} \\
(1.78)\end{array}$ \\
\hline 4 & Poultry manure @ 1 ton/ha & $\begin{array}{l}5.03^{a b c} \\
(0.77)\end{array}$ & $\begin{array}{l}13.74^{c} \\
(5.65)\end{array}$ & $\begin{array}{l}17.05^{\text {de }} \\
(8.62)\end{array}$ & $\begin{array}{l}19.26^{\mathrm{cd}} \\
(10.88)\end{array}$ & $\begin{array}{l}13.77^{\text {de }} \\
(5.67)\end{array}$ \\
\hline 5 & $\begin{array}{l}\text { Poultry manure @ } 1 \text { ton/ha + B. bassiana 1.15 WP } \\
\left(1 \times 10^{8} \mathrm{cfu} / \mathrm{g}\right) @ 2 \mathrm{~kg} / \mathrm{ha}\end{array}$ & $\begin{array}{l}5.34^{\mathrm{ab}} \\
(0.87)\end{array}$ & $\begin{array}{l}14.32^{c} \\
(6.12)\end{array}$ & $\begin{array}{l}15.41^{\mathrm{e}} \\
(7.11)\end{array}$ & $\begin{array}{l}18.11^{d} \\
(9.69)\end{array}$ & $\begin{array}{l}13.29^{e} \\
(5.28)\end{array}$ \\
\hline 6 & $\begin{array}{l}\text { Poultry manure @ } 1 \text { ton/ha }+ \text { M. anisopliae 1.15 WP } \\
\left(1 \times 10^{8} \mathrm{cfu} / \mathrm{g}\right) @ 2 \mathrm{~kg} / \mathrm{ha}\end{array}$ & $\begin{array}{l}5.25^{\mathrm{ab}} \\
(0.84)\end{array}$ & $\begin{array}{l}11.22^{d} \\
(3.83)\end{array}$ & $\begin{array}{l}11.79^{f} \\
(4.33)\end{array}$ & $\begin{array}{l}14.97^{\mathrm{e}} \\
(6.68)\end{array}$ & $\begin{array}{l}10.80^{f} \\
(3.51)\end{array}$ \\
\hline 7 & Vermicompost @ 1 ton/ha & $\begin{array}{l}5.34^{\mathrm{ab}} \\
(0.87)\end{array}$ & $\begin{array}{l}17.50^{\mathrm{ab}} \\
(9.06)\end{array}$ & $\begin{array}{l}21.08^{\mathrm{ab}} \\
(12.95)\end{array}$ & $\begin{array}{l}22.88^{b} \\
(15.19)\end{array}$ & $\begin{array}{l}16.70^{\mathrm{b}} \\
(8.26)\end{array}$ \\
\hline 8 & $\begin{array}{l}\text { Vermicompost @ } 1 \text { ton/ha + B. bassiana 1.15 WP } \\
\left(1 \times 10^{8} \mathrm{cfu} / \mathrm{g}\right) @ 2 \mathrm{~kg} / \mathrm{ha}\end{array}$ & $\begin{array}{l}3.70^{\text {de }} \\
(0.42)\end{array}$ & $\begin{array}{l}16.0^{\mathrm{bc}} \\
(7.63)\end{array}$ & $\begin{array}{l}18.69^{\mathrm{cd}} \\
(10.28)\end{array}$ & $\begin{array}{l}21.01^{b c} \\
(12.96)\end{array}$ & $\begin{array}{l}14.85^{\mathrm{cd}} \\
(6.57)\end{array}$ \\
\hline 9 & $\begin{array}{l}\text { Vermicompost @ } 1 \text { ton/ha + M. anisopliae 1.15 WP } \\
\left(1 \times 10^{8} \mathrm{cfu} / \mathrm{g}\right) @ 2 \mathrm{~kg} / \mathrm{ha}\end{array}$ & $\begin{array}{l}1.12^{f} \\
(0.04)\end{array}$ & $\begin{array}{l}6.54^{f} \\
(1.33)\end{array}$ & $\begin{array}{l}7.79^{h} \\
(1.93)\end{array}$ & $\begin{array}{l}9.52^{f} \\
(2.76)\end{array}$ & $\begin{array}{l}6.24^{\mathrm{i}} \\
(1.18)\end{array}$ \\
\hline 10 & Castor cake @ 1 ton/ha & $\begin{array}{l}3.31^{e} \\
(0.34)\end{array}$ & $\begin{array}{l}17.21^{\mathrm{ab}} \\
(8.77)\end{array}$ & $\begin{array}{l}19.41^{b c} \\
(11.06)\end{array}$ & $\begin{array}{l}21.46^{b c} \\
(13.46)\end{array}$ & $\begin{array}{l}15.34^{c} \\
(7.00)\end{array}$ \\
\hline 11 & $\begin{array}{l}\text { Castor cake @ } 1 \text { ton/ha + B. bassiana } 1.15 \text { WP }\left(1 \times 10^{8} \mathrm{cfu} / \mathrm{g}\right) \\
@ 2 \mathrm{~kg} / \mathrm{ha}\end{array}$ & $\begin{array}{l}5.03^{\mathrm{abc}} \\
(0.77)\end{array}$ & $\begin{array}{l}14.66^{c} \\
(6.43)\end{array}$ & $\begin{array}{l}16.12^{\mathrm{e}} \\
(7.73)\end{array}$ & $\begin{array}{l}19.34^{\mathrm{cd}} \\
(11.04)\end{array}$ & $\begin{array}{l}13.78^{\text {de }} \\
(5.67)\end{array}$ \\
\hline 12 & $\begin{array}{l}\text { Castor cake @ } 1 \text { ton/ha + M. anisopliae 1.15WP }\left(1 \times 10^{8} \mathrm{cfu} / \mathrm{g}\right) \\
\text { @ } 2 \text { kg/ha }\end{array}$ & $\begin{array}{l}4.18^{\mathrm{cd}} \\
(0.33)\end{array}$ & $\begin{array}{l}6.59^{f} \\
(1.45)\end{array}$ & $\begin{array}{l}8.35^{g h} \\
(2.13)\end{array}$ & $\begin{array}{l}9.66^{\mathrm{f}} \\
(2.85)\end{array}$ & $\begin{array}{l}6.94^{\mathrm{hi}} \\
(1.46)\end{array}$ \\
\hline 13 & Neem cake@ @ ton/ha & $\begin{array}{l}5.72^{a} \\
(0.66)\end{array}$ & $\begin{array}{l}19.07^{\mathrm{a}} \\
(10.69)\end{array}$ & $\begin{array}{l}21.66^{a} \\
(13.66)\end{array}$ & $\begin{array}{l}26.85^{a} \\
(20.45)\end{array}$ & $\begin{array}{l}18.32^{a} \\
(9.88)\end{array}$ \\
\hline \multirow[t]{3}{*}{ S.Em. \pm} & $\mathrm{T}$ & 0.28 & 0.75 & 0.73 & 0.74 & 0.395 \\
\hline & $\mathrm{P}$ & - & - & - & - & 0.219 \\
\hline & $\mathrm{T} \times \mathrm{P}$ & - & - & - & - & 0.790 \\
\hline C.D. at $5 \%$ & $\mathrm{~T}$ & NS & 2.18 & 2.22 & 2.17 & 1.108 \\
\hline C.V. (\%) & & 10.70 & 10.39 & 10.23 & 11.12 & 11.36 \\
\hline
\end{tabular}

1. Figures in parentheses are retransformed values of arc sin transformation; DAG: Days after germination; 2 . Treatment means with the letter(s) in common are not significant by DNMRT at $5 \%$ level of significance; 3 . Significant parameters and their interactions: T, P, T $\times P$ 
Table 3 Effect of entomopathogenic fungi enriched with organic amendments against white grub in groundnut (season of 2019)

\begin{tabular}{|c|c|c|c|c|c|c|}
\hline \multirow[t]{2}{*}{ Tr. no } & \multirow[t]{2}{*}{ Treatments } & \multicolumn{5}{|c|}{ Dead plants (\%) } \\
\hline & & 1-20 DAG & 21-40 DAG & $41-60$ DAG & $61-80$ DAG & Mean over period \\
\hline 1 & Neem cake@ 1 ton/ha & $\begin{array}{l}5.03^{\mathrm{ab}} \\
(0.77)\end{array}$ & $\begin{array}{l}14.21^{b} \\
(6.03)\end{array}$ & $\begin{array}{l}16.84^{\text {cde }} \\
(8.42)\end{array}$ & $\begin{array}{l}21.16^{\mathrm{b}} \\
(13.05)\end{array}$ & $\begin{array}{l}14.30^{c} \\
(6.10)\end{array}$ \\
\hline 2 & $\begin{array}{l}\text { Neem cake @ } 1 \text { ton/ha + Beauveria bassiana 1.15 WP } \\
\left(1 \times 10^{8} \mathrm{cfu} / \mathrm{g}\right) @ 2 \mathrm{~kg} / \mathrm{ha}\end{array}$ & $\begin{array}{l}4.95^{\mathrm{ab}} \\
(0.75)\end{array}$ & $\begin{array}{l}13.02^{b} \\
(5.08)\end{array}$ & $\begin{array}{l}14.05^{\mathrm{ef}} \\
(5.92)\end{array}$ & $\begin{array}{l}17.44^{c} \\
(9.00)\end{array}$ & $\begin{array}{l}12.36^{d} \\
(4.58)\end{array}$ \\
\hline 3 & $\begin{array}{l}\text { Neem cake @ } 1 \text { ton/ha + Metarhizium anisopliae 1.15 WP } \\
\left(1 \times 10^{8} \mathrm{cfu} / \mathrm{g}\right) @ 2 \mathrm{~kg} / \mathrm{ha}\end{array}$ & $\begin{array}{l}4.90^{\mathrm{ab}} \\
(0.73)\end{array}$ & $\begin{array}{l}9.13^{c} \\
(2.53)\end{array}$ & $\begin{array}{l}9.87^{9} \\
(3.00)\end{array}$ & $\begin{array}{l}12.68^{d} \\
(5.07)\end{array}$ & $\begin{array}{l}9.14^{e} \\
(2.52)\end{array}$ \\
\hline 4 & Poultry manure@ @ ton/ha & $\begin{array}{l}5.03^{\mathrm{ab}} \\
(0.77)\end{array}$ & $\begin{array}{l}13.70^{b} \\
(5.62)\end{array}$ & $\begin{array}{l}17.09^{b c d} \\
(8.64)\end{array}$ & $\begin{array}{l}21.46^{b} \\
(13.39)\end{array}$ & $\begin{array}{l}14.31^{\mathrm{c}} \\
(6.11)\end{array}$ \\
\hline 5 & $\begin{array}{l}\text { Poultry manure @ } 1 \text { ton/ha + B. bassiana 1.15WP } \\
\left(1 \times 10^{8} \mathrm{cfu} / \mathrm{g}\right) @ 2 \mathrm{~kg} / \mathrm{ha}\end{array}$ & $\begin{array}{l}5.25^{\mathrm{a}} \\
(0.84)\end{array}$ & $\begin{array}{l}14.04^{b} \\
(5.89)\end{array}$ & $\begin{array}{l}16.66^{\text {de }} \\
(8.33)\end{array}$ & $\begin{array}{l}21.00^{b} \\
(12.89)\end{array}$ & $\begin{array}{l}14.23^{c} \\
(6.04)\end{array}$ \\
\hline 6 & $\begin{array}{l}\text { Poultry manure @ } 1 \text { ton/ha + M. anisopliae 1.15 WP } \\
\left(1 \times 10^{8} \mathrm{cfu} / \mathrm{g}\right) @ 2 \mathrm{~kg} / \mathrm{ha}\end{array}$ & $\begin{array}{l}4.52^{\mathrm{ab}} \\
(0.63)\end{array}$ & $\begin{array}{l}12.81^{\mathrm{b}} \\
(4.95)\end{array}$ & $\begin{array}{l}13.63^{f} \\
(5.67)\end{array}$ & $\begin{array}{l}17.15^{c} \\
(8.72)\end{array}$ & $\begin{array}{l}12.02^{d} \\
(4.34)\end{array}$ \\
\hline 7 & Vermicompost @ 1 ton/ha & $\begin{array}{l}5.35^{\mathrm{a}} \\
(0.87)\end{array}$ & $\begin{array}{l}15.58^{\mathrm{ab}} \\
(7.25)\end{array}$ & $\begin{array}{l}20.26^{\mathrm{a}} \\
(12.00)\end{array}$ & $\begin{array}{l}24.23^{\mathrm{ab}} \\
(16.92)\end{array}$ & $\begin{array}{l}16.35^{b} \\
(7.92)\end{array}$ \\
\hline 8 & $\begin{array}{l}\text { Vermicompost @ } 1 \text { ton/ha + B. bassiana 1.15WP } \\
\left(1 \times 10^{8} \mathrm{cfu} / \mathrm{g}\right) @ 2 \mathrm{~kg} / \mathrm{ha}\end{array}$ & $\begin{array}{l}4.51^{\mathrm{ab}} \\
(0.62)\end{array}$ & $\begin{array}{l}14.88^{\mathrm{ab}} \\
(6.66)\end{array}$ & $\begin{array}{l}19.59^{\mathrm{abc}} \\
(11.25)\end{array}$ & $\begin{array}{l}23.91^{\mathrm{ab}} \\
(16.50)\end{array}$ & $\begin{array}{l}15.72^{b} \\
(7.34)\end{array}$ \\
\hline 9 & $\begin{array}{l}\text { Vermicompost @ } 1 \text { ton/ha + M. anisopliae 1.15WP } \\
\left(1 \times 10^{8} \mathrm{cfu} / \mathrm{g}\right) @ 2 \mathrm{~kg} / \mathrm{ha}\end{array}$ & $\begin{array}{l}4.03^{b} \\
(0.50)\end{array}$ & $\begin{array}{l}8.46^{c} \\
(2.20)\end{array}$ & $\begin{array}{l}9.14^{\mathrm{g}} \\
(2.53)\end{array}$ & $\begin{array}{l}11.63^{d} \\
(4.10)\end{array}$ & $\begin{array}{l}8.31^{\mathrm{e}} \\
(2.09)\end{array}$ \\
\hline 10 & Castor cake @ 1 ton/ha & $\begin{array}{l}4.43^{\mathrm{ab}} \\
(0.63)\end{array}$ & $\begin{array}{l}15.45^{\mathrm{ab}} \\
(7.17)\end{array}$ & $\begin{array}{l}19.86^{\mathrm{ab}} \\
(11.55)\end{array}$ & $\begin{array}{l}23.99^{\mathrm{ab}} \\
(16.67)\end{array}$ & $\begin{array}{l}15.93^{b} \\
(7.53)\end{array}$ \\
\hline 11 & $\begin{array}{l}\text { Castor cake @ } 1 \text { ton/ha + B. bassiana } 1.15 \text { WP }\left(1 \times 10^{8} \mathrm{cfu} / \mathrm{g}\right) \\
\text { @ } 2 \mathrm{~kg} / \mathrm{ha}\end{array}$ & $\begin{array}{l}5.15^{\mathrm{a}} \\
(0.81)\end{array}$ & $\begin{array}{l}13.02^{b} \\
(5.17)\end{array}$ & $\begin{array}{l}14.49^{\text {def }} \\
(6.33)\end{array}$ & $\begin{array}{l}17.62^{c} \\
(9.17)\end{array}$ & $\begin{array}{l}12.56^{d} \\
(4.73)\end{array}$ \\
\hline 12 & $\begin{array}{l}\text { Castor cake @ } 1 \text { ton/ha + M. anisopliae } 1.15 \text { WP }\left(1 \times 10^{8} \mathrm{cfu} / \mathrm{g}\right) \\
\text { @ } 2 \mathrm{~kg} / \mathrm{ha}\end{array}$ & $\begin{array}{l}4.46^{\mathrm{ab}} \\
(0.61)\end{array}$ & $\begin{array}{l}8.86^{c} \\
(2.48)\end{array}$ & $\begin{array}{l}9.44^{\mathrm{g}} \\
(2.77)\end{array}$ & $\begin{array}{l}12.36^{d} \\
(4.67)\end{array}$ & $\begin{array}{l}8.78^{\mathrm{e}} \\
(2.33)\end{array}$ \\
\hline 13 & Neem cake@ 1 ton/ha & $\begin{array}{l}5.02^{\mathrm{ab}} \\
(0.77)\end{array}$ & $\begin{array}{l}17.42^{\mathrm{a}} \\
(9.00)\end{array}$ & $\begin{array}{l}22.14^{\mathrm{a}} \\
(14.22)\end{array}$ & $\begin{array}{l}27.05^{\mathrm{a}} \\
(20.78)\end{array}$ & $\begin{array}{l}17.90^{\mathrm{a}} \\
(9.45)\end{array}$ \\
\hline \multirow[t]{3}{*}{ S.Em. \pm} & $\mathrm{T}$ & 0.31 & 0.83 & 0.93 & 1.12 & 0.435 \\
\hline & P & - & - & - & - & 0.241 \\
\hline & $T \times P$ & - & - & - & - & 0.871 \\
\hline C. D. at $5 \%$ & $\mathrm{~T}$ & NS & 2.41 & 2.71 & 3.28 & 1.221 \\
\hline C.V. (\%) & & 10.98 & 10.91 & 10.29 & 10.04 & 11.40 \\
\hline
\end{tabular}

1. Figures in parentheses are retransformed values of arc sin transformation; DAG: Days after germination; 2 . Treatment means with the letter(s) in common are not significant by DNMRT at $5 \%$ level of significance; 3 . Significant parameters and their interactions: T, P, T $\times P$

1.15 WP $\left(1 \times 10^{8} \mathrm{cfu} / \mathrm{g}\right) @ 2 \mathrm{~kg} / \mathrm{ha}$, neem cake @ 1 ton/ ha + M. anisopliae 1.15 WP $\left(1 \times 10^{8} \mathrm{cfu} / \mathrm{g}\right) @ 2 \mathrm{~kg} /$ ha and neem cake@1 ton/ha + B. bassiana 1.15 WP $\left(1 \times 10^{8} \mathrm{cfu} / \mathrm{g}\right) @ 2 \mathrm{~kg} / \mathrm{ha}$, whereas, at 60 and 80 DAG, minimum plant mortality was observed in the treatment with vermicompost @ 1 ton/ha $+M$. anisopliae 1.15 WP $\left(1 \times 10^{8} \mathrm{cfu} / \mathrm{g}\right) @ 2 \mathrm{~kg} / \mathrm{ha}$ and it was at part with Castor cake@ 1 ton/ha + M. anisopliae 1.15 WP $\left(1 \times 10^{8} \mathrm{cfu} / \mathrm{g}\right) @ 2 \mathrm{~kg} / \mathrm{ha}$ and neem cake@ $1 \mathrm{ton} /$ ha $+M$. anisopliae 1.15 WP $\left(1 \times 10^{8} \mathrm{cfu} / \mathrm{g}\right) @ 2 \mathrm{~kg} /$ ha. Based on pooled results of season 2018, the most effective treatment was vermicompost @ 1 ton/ha $+M$. anisopliae 1.15 WP $\left(1 \times 10^{8} \mathrm{cfu} / \mathrm{g}\right) @ 2 \mathrm{~kg} / \mathrm{ha}$ and it was at part with castor cake@ 1 ton/ha $+M$. anisopliae 1.15 WP $\left(1 \times 10^{8} \mathrm{cfu} / \mathrm{g}\right) @ 2 \mathrm{~kg} / \mathrm{ha}$ with minimum plant mortality 1.18 and $1.46 \%$, respectively. In the season 2019 (Table 3), at 40, 60 and 80 days after germination the minimum plant mortality was observed in the plot treated with soil application of vermicompost @ 1 ton/ ha $+M$. anisopliae 1.15 WP $\left(1 \times 10^{8} \mathrm{cfu} / \mathrm{g}\right) @ 2 \mathrm{~kg} / \mathrm{ha}$ and it was at part with castor cake@ 1 ton/ha $+M$. anisopliae 1.15 WP $\left(1 \times 10^{8} \mathrm{cfu} / \mathrm{g}\right) @ 2 \mathrm{~kg} / \mathrm{ha}$ and neem cake @ 1 ton $/$ ha + M. anisopliae 1.15 WP $\left(1 \times 10^{8} \mathrm{cfu} / \mathrm{g}\right)$ @ $2 \mathrm{~kg} / \mathrm{ha}$, whereas, based on pooled results of season 2019, the most effective treatment was vermicompost @ 1 ton/ha + M. anisopliae 1.15 WP $\left(1 \times 10^{8} \mathrm{cfu} / \mathrm{g}\right) @$ $2 \mathrm{~kg} / \mathrm{ha}(2.09 \%)$ and it was at part with castor cake @ 1 ton/ha + M. anisopliae 1.15 WP $\left(1 \times 10^{8} \mathrm{cfu} / \mathrm{g}\right) @ 2 \mathrm{~kg} /$ ha $(2.33 \%)$ and neem cake @ 1 ton/ha $+M$. anisopliae $1.15 \mathrm{WP}\left(1 \times 10^{8} \mathrm{cfu} / \mathrm{g}\right) @ 2 \mathrm{~kg} / \mathrm{ha}(2.52 \%)$. The results of season 2020 (Table 4), at 40, 60 and 80 days after germination showed that the minimum plant mortality was observed in the treatment of vermicompost @ 1 ton/ha + M. anisopliae $1.15 \mathrm{WP}\left(1 \times 10^{8} \mathrm{cfu} / \mathrm{g}\right) @ 2 \mathrm{~kg} /$ ha and it was at part with castor cake @ 1 ton/ha $+M$. 
Table 4 Effect of entomopathogenic fungi enriched with organic amendments against white grub in groundnut (season of 2020)

\begin{tabular}{|c|c|c|c|c|c|c|}
\hline \multirow[t]{2}{*}{ Tr. no } & \multirow[t]{2}{*}{ Treatments } & \multicolumn{5}{|c|}{ Dead plants (\%) } \\
\hline & & 1-20 DAG & 21-40 DAG & $41-60$ DAG & $61-80$ DAG & Mean over period \\
\hline 1 & Neem cake@ 1 ton/ha & $\begin{array}{l}5.29^{\mathrm{a}} \\
(0.85)\end{array}$ & $\begin{array}{l}14.30^{b} \\
(6.10)\end{array}$ & $\begin{array}{l}17.06^{\mathrm{b}} \\
(8.61)\end{array}$ & $\begin{array}{l}21.30^{\mathrm{bc}} \\
(13.20)\end{array}$ & $\begin{array}{l}14.48^{c} \\
(6.25)\end{array}$ \\
\hline 2 & $\begin{array}{l}\text { Neem cake @ } 1 \text { ton/ha + Beauveria bassiana 1.15 WP } \\
\left(1 \times 10^{8} \mathrm{cfu} / \mathrm{g}\right) @ 2 \mathrm{~kg} / \mathrm{ha}\end{array}$ & $\begin{array}{l}5.22^{\mathrm{a}} \\
(0.83)\end{array}$ & $\begin{array}{l}13.02^{b} \\
(5.08)\end{array}$ & $\begin{array}{l}14.28^{\mathrm{cd}} \\
(6.08)\end{array}$ & $\begin{array}{l}17.81^{c d} \\
(9.36)\end{array}$ & $\begin{array}{l}12.55^{d} \\
(4.72)\end{array}$ \\
\hline 3 & $\begin{array}{l}\text { Neem cake @ } 1 \text { ton/ha + Metarhizium anisopliae 1.15 WP } \\
\left(1 \times 10^{8} \mathrm{cfu} / \mathrm{g}\right) @ 2 \mathrm{~kg} / \mathrm{ha}\end{array}$ & $\begin{array}{l}5.09^{\mathrm{a}} \\
(0.79)\end{array}$ & $\begin{array}{l}8.90^{c} \\
(2.40)\end{array}$ & $\begin{array}{l}9.65^{e} \\
(2.81)\end{array}$ & $\begin{array}{l}12.42^{\mathrm{e}} \\
(4.63)\end{array}$ & $\begin{array}{l}9.01^{\mathrm{e}} \\
(2.45)\end{array}$ \\
\hline 4 & Poultry manure@ @ ton/ha & $\begin{array}{l}5.10^{\mathrm{a}} \\
(0.79)\end{array}$ & $\begin{array}{l}13.86^{b} \\
(5.74)\end{array}$ & $\begin{array}{l}17.20^{b} \\
(8.74)\end{array}$ & $\begin{array}{l}21.74^{\mathrm{b}} \\
(13.72)\end{array}$ & $\begin{array}{l}14.47^{c} \\
(6.24)\end{array}$ \\
\hline 5 & $\begin{array}{l}\text { Poultry manure @ } 1 \text { ton/ha + B. bassiana 1.15WP } \\
\left(1 \times 10^{8} \mathrm{cfu} / \mathrm{g}\right) @ 2 \mathrm{~kg} / \mathrm{ha}\end{array}$ & $\begin{array}{l}5.10^{\mathrm{a}} \\
(0.79)\end{array}$ & $\begin{array}{l}14.14^{b} \\
(5.97)\end{array}$ & $\begin{array}{l}16.92^{b c} \\
(8.47)\end{array}$ & $\begin{array}{l}21.11^{b c} \\
(12.97)\end{array}$ & $\begin{array}{l}14.31^{\mathrm{c}} \\
(6.11)\end{array}$ \\
\hline 6 & $\begin{array}{l}\text { Poultry manure @ } 1 \text { ton/ha + M. anisopliae 1.15 WP } \\
\left(1 \times 10^{8} \mathrm{cfu} / \mathrm{g}\right) @ 2 \mathrm{~kg} / \mathrm{ha}\end{array}$ & $\begin{array}{l}4.83^{\mathrm{ab}} \\
(0.71)\end{array}$ & $\begin{array}{l}12.98^{\mathrm{b}} \\
(5.05)\end{array}$ & $\begin{array}{l}14.11^{d} \\
(5.94)\end{array}$ & $\begin{array}{l}17.33^{d} \\
(8.87)\end{array}$ & $\begin{array}{l}12.31^{d} \\
(4.55)\end{array}$ \\
\hline 7 & Vermicompost @ 1 ton/ha & $\begin{array}{l}5.21^{\mathrm{a}} \\
(0.82)\end{array}$ & $\begin{array}{l}15.41^{\mathrm{ab}} \\
(7.06)\end{array}$ & $\begin{array}{l}20.32^{\mathrm{a}} \\
(12.06)\end{array}$ & $\begin{array}{l}24.23^{\mathrm{ab}} \\
(16.85)\end{array}$ & $\begin{array}{l}16.28^{b} \\
(7.86)\end{array}$ \\
\hline 8 & $\begin{array}{l}\text { Vermicompost @ } 1 \text { ton/ha + B. bassiana 1.15WP } \\
\left(1 \times 10^{8} \mathrm{cfu} / \mathrm{g}\right) @ 2 \mathrm{~kg} / \mathrm{ha}\end{array}$ & $\begin{array}{l}4.70^{\mathrm{ab}} \\
(0.67)\end{array}$ & $\begin{array}{l}15.09^{\mathrm{ab}} \\
(6.78)\end{array}$ & $\begin{array}{l}19.85^{\mathrm{a}} \\
(11.52)\end{array}$ & $\begin{array}{l}24.02^{\mathrm{ab}} \\
(16.57)\end{array}$ & $\begin{array}{l}15.91^{b} \\
(7.51)\end{array}$ \\
\hline 9 & $\begin{array}{l}\text { Vermicompost @ } 1 \text { ton/ha + M. anisopliae 1.15WP } \\
\left(1 \times 10^{8} \mathrm{cfu} / \mathrm{g}\right) @ 2 \mathrm{~kg} / \mathrm{ha}\end{array}$ & $\begin{array}{l}4.25^{b} \\
(0.55)\end{array}$ & $\begin{array}{l}8.22^{c} \\
(2.05)\end{array}$ & $\begin{array}{l}8.71^{\mathrm{e}} \\
(2.29)\end{array}$ & $\begin{array}{l}11.45^{\mathrm{e}} \\
(3.94)\end{array}$ & $\begin{array}{l}8.15^{\mathrm{e}} \\
(2.01)\end{array}$ \\
\hline 10 & Castor cake @ 1 ton/ha & $\begin{array}{l}4.70^{\mathrm{ab}} \\
(0.67)\end{array}$ & $\begin{array}{l}15.55^{\mathrm{ab}} \\
(7.19)\end{array}$ & $\begin{array}{l}20.06^{\mathrm{a}} \\
(11.75)\end{array}$ & $\begin{array}{l}24.23^{\mathrm{ab}} \\
(16.84)\end{array}$ & $\begin{array}{l}16.13^{b} \\
(7.72)\end{array}$ \\
\hline 11 & $\begin{array}{l}\text { Castor cake @ } 1 \text { ton/ha + B. bassiana } 1.15 \text { WP }\left(1 \times 10^{8} \mathrm{cfu} / \mathrm{g}\right) \\
\text { @ } 2 \mathrm{~kg} / \mathrm{ha}\end{array}$ & $\begin{array}{l}5.34^{\mathrm{a}} \\
(0.87)\end{array}$ & $\begin{array}{l}13.06^{b} \\
(5.11)\end{array}$ & $\begin{array}{l}14.95^{\mathrm{bcd}} \\
(6.65)\end{array}$ & $\begin{array}{l}17.95^{\mathrm{cd}} \\
(9.50)\end{array}$ & $\begin{array}{l}12.82^{d} \\
(4.92)\end{array}$ \\
\hline 12 & $\begin{array}{l}\text { Castor cake @ } 1 \text { ton/ha + M. anisopliae } 1.15 \text { WP }\left(1 \times 10^{8} \mathrm{cfu} / \mathrm{g}\right) \\
\text { @ } 2 \mathrm{~kg} / \mathrm{ha}\end{array}$ & $\begin{array}{l}4.73^{\mathrm{ab}} \\
(0.68)\end{array}$ & $\begin{array}{l}8.51^{c} \\
(2.19)\end{array}$ & $\begin{array}{l}9.14^{\mathrm{e}} \\
(2.53)\end{array}$ & $\begin{array}{l}12.28^{\mathrm{e}} \\
(4.52)\end{array}$ & $\begin{array}{l}8.66^{\mathrm{e}} \\
(2.27)\end{array}$ \\
\hline 13 & Neem cake@ 1 ton/ha & $\begin{array}{l}5.10^{\mathrm{a}} \\
(0.79)\end{array}$ & $\begin{array}{l}17.53^{\mathrm{a}} \\
(9.07)\end{array}$ & $\begin{array}{l}22.44^{\mathrm{a}} \\
(14.57)\end{array}$ & $\begin{array}{l}27.30^{\mathrm{a}} \\
(21.03)\end{array}$ & $\begin{array}{l}18.09^{\mathrm{a}} \\
(9.64)\end{array}$ \\
\hline \multirow[t]{3}{*}{ S.Em. \pm} & $\mathrm{T}$ & 0.25 & 0.80 & 0.86 & 1.14 & 0.427 \\
\hline & P & - & - & - & - & 0.237 \\
\hline & $T \times P$ & - & - & - & - & 0.854 \\
\hline C. D. at $5 \%$ & $\mathrm{~T}$ & NS & 2.34 & 2.50 & 3.34 & 1.198 \\
\hline C.V. (\%) & & 8.67 & 10.59 & 9.42 & 10.18 & 11.10 \\
\hline
\end{tabular}

1. Figures in parentheses are retransformed values of arc sin transformation; DAG: Days after germination; 2 . treatment means with the letter(s) in common are not significant by DNMRT at $5 \%$ level of significance; 3 . significant parameters and their interactions: $T, P, T \times P$

anisopliae 1.15 WP $\left(1 \times 10^{8} \mathrm{cfu} / \mathrm{g}\right) @ 2 \mathrm{~kg} / \mathrm{ha}$ and neem cake@ 1 ton/ha + M. anisopliae 1.15 WP $\left(1 \times 10^{8} \mathrm{cfu} / \mathrm{g}\right)$ @ $2 \mathrm{~kg} / \mathrm{ha}$. However, based on pooled results of season 2020, vermicompost@ 1 ton/ha $+M$. anisopliae 1.15 WP $\left(1 \times 10^{8} \mathrm{cfu} / \mathrm{g}\right) @ 2 \mathrm{~kg} / \mathrm{ha}$ was found most effective treatment which was at part with castor cake @ 1 ton/ha + M. anisopliae $1.15 \mathrm{WP}\left(1 \times 10^{8} \mathrm{cfu} / \mathrm{g}\right) @ 2 \mathrm{~kg} /$ ha and neem cake @ 1 ton/ha + M. anisopliae 1.15 WP $\left(1 \times 10^{8} \mathrm{cfu} / \mathrm{g}\right) @ 2 \mathrm{~kg} / \mathrm{ha}$ with minimum plant mortality $2.01,2.27$ and $2.45 \%$, respectively.

The results presented in Table 5 revealed that during season 2018, the treatment vermicompost @ 1 ton/ ha + M. anisopliae 1.15 WP $\left(1 \times 10^{8} \mathrm{cfu} / \mathrm{g}\right) @ 2 \mathrm{~kg} / \mathrm{ha}$ was found most effective against white grub and it was at par with castor cake @ 1 ton/ha + M. anisopliae 1.15 WP $\left(1 \times 10^{8} \mathrm{cfu} / \mathrm{g}\right) @ 2 \mathrm{~kg} / \mathrm{ha}$, whereas, the results of seasons 2019 and 2020 showed that minimum plant mortality was observed in the treatment of vermicompost @ 1 ton/ha + M. anisopliae 1.15 WP $\left(1 \times 10^{8} \mathrm{cfu} / \mathrm{g}\right)$ @ $2 \mathrm{~kg} / \mathrm{ha}$ and it was at part with castor cake @ $1 \mathrm{ton} /$ ha + M. anisopliae 1.15 WP $\left(1 \times 10^{8} \mathrm{cfu} / \mathrm{g}\right) @ 2 \mathrm{~kg} / \mathrm{ha}$ and neem cake@ 1 ton/ha + M. anisopliae 1.15 WP $\left(1 \times 10^{8} \mathrm{cfu} / \mathrm{g}\right) @ 2 \mathrm{~kg} / \mathrm{ha}$.

Looking to overall pooled results of the 3 consecutive seasons 2018, 2019 and 2020, it is clearly indicated that minimum plant mortality due to white grub was recorded in the plot treated with soil application of vermicompost @ 1 ton/ha $+M$. anisopliae 1.15 WP $\left(1 \times 10^{8} \mathrm{cfu} / \mathrm{g}\right)$ @ $2 \mathrm{~kg} / \mathrm{ha}$ and it was at part with castor cake @ 1 ton/ ha $+M$. anisopliae $1.15 \mathrm{WP}\left(1 \times 10^{8} \mathrm{cfu} / \mathrm{g}\right) @ 2 \mathrm{~kg} /$ ha and neem cake@ 1 ton/ha $+M$. anisopliae 1.15 WP $\left(1 \times 10^{8} \mathrm{cfu} / \mathrm{g}\right) @ 2 \mathrm{~kg} / \mathrm{ha}$ which show only $1.74,2.00$ and $2.24 \%$ plant damage, respectively (Table 5 ).

The results on cumulative plant damage due to white grub during different seasons clearly showed that soil 
Table 5 Effect of entomopathogenic fungi enriched with organic amendments against white grub in groundnut (pooled over period and seasons)

\begin{tabular}{|c|c|c|c|c|c|}
\hline \multirow[t]{2}{*}{ Tr. no } & \multirow[t]{2}{*}{ Treatments } & \multicolumn{3}{|c|}{ Dead plants (\%) } & \multirow{2}{*}{$\begin{array}{l}\text { Pooled over } \\
\text { period and } \\
\text { seasons }\end{array}$} \\
\hline & & 2018 & 2019 & 2020 & \\
\hline 1 & Neem cake@ @ ton/ha & $\begin{array}{l}9.77^{\mathrm{fg}} \\
(2.88)\end{array}$ & $\begin{array}{l}14.30^{c} \\
(6.10)\end{array}$ & $\begin{array}{l}14.48^{c} \\
(6.25)\end{array}$ & $\begin{array}{l}12.85^{\text {def }} \\
(4.95)\end{array}$ \\
\hline 2 & Neem cake@1 ton/ha+Beauveria bassiana 1.15WP (1×108 cfu/g)@ 2kg/ha & $\begin{array}{l}9.179 \\
(2.54)\end{array}$ & $\begin{array}{l}12.36^{d} \\
(4.58)\end{array}$ & $\begin{array}{l}12.55^{\mathrm{d}} \\
(4.72)\end{array}$ & $\begin{array}{l}11.36^{\mathrm{f}} \\
(3.88)\end{array}$ \\
\hline 3 & Neem cake@ 1 ton/ha+Metarhizium anisopliae 1.15WP (1×108 cfu/g)@ 2 kg/ha & $\begin{array}{l}7.66^{h} \\
(1.78)\end{array}$ & $\begin{array}{l}9.14^{\mathrm{e}} \\
(2.52)\end{array}$ & $\begin{array}{l}9.01^{\mathrm{e}} \\
(2.45)\end{array}$ & $\begin{array}{l}8.60^{9} \\
(2.24)\end{array}$ \\
\hline 4 & Poultry manure @ 1 ton/ha & $\begin{array}{l}13.77^{\text {de }} \\
(5.67)\end{array}$ & $\begin{array}{l}14.31^{\mathrm{c}} \\
(6.11)\end{array}$ & $\begin{array}{l}14.47^{c} \\
(6.24)\end{array}$ & $\begin{array}{l}14.18^{\mathrm{cd}} \\
(6.00)\end{array}$ \\
\hline 5 & Poultry manure@ 1 ton/ha+B.bassiana 1.15 WP $\left(1 \times 10^{8} \mathrm{cfu} / \mathrm{g}\right) @ 2$ kg/ha & $\begin{array}{l}13.29^{\mathrm{e}} \\
(5.28)\end{array}$ & $\begin{array}{l}14.23^{c} \\
(6.04)\end{array}$ & $\begin{array}{l}14.31^{c} \\
(6.11)\end{array}$ & $\begin{array}{l}13.94^{\mathrm{d}} \\
(5.80)\end{array}$ \\
\hline 6 & 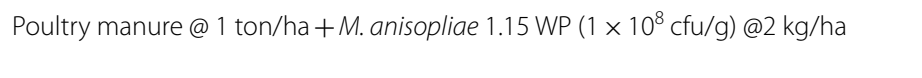 & $\begin{array}{l}10.80^{f} \\
(3.51)\end{array}$ & $\begin{array}{l}12.02^{d} \\
(4.34)\end{array}$ & $\begin{array}{l}12.31^{d} \\
(4.55)\end{array}$ & $\begin{array}{l}11.71^{\mathrm{ef}} \\
(4.12)\end{array}$ \\
\hline 7 & Vermicompost @ 1 ton/ha & $\begin{array}{l}16.70^{\mathrm{b}} \\
(8.26)\end{array}$ & $\begin{array}{l}16.35^{b} \\
(7.92)\end{array}$ & $\begin{array}{l}16.28^{b} \\
(7.86)\end{array}$ & $\begin{array}{l}16.44^{\mathrm{b}} \\
(8.01)\end{array}$ \\
\hline 8 & Vermicompost @ 1 ton/ha+B.bassiana 1.15 WP $\left(1 \times 10^{8} \mathrm{cfu} / \mathrm{g}\right) @ 2$ kg/ha & $\begin{array}{l}14.85^{\mathrm{cd}} \\
(6.57)\end{array}$ & $\begin{array}{l}15.72^{b} \\
(7.34)\end{array}$ & $\begin{array}{l}15.91^{b} \\
(7.51)\end{array}$ & $\begin{array}{l}15.49^{b c} \\
(7.13)\end{array}$ \\
\hline 9 & Vermicompost @ 1 ton/ha+M. anisopliae 1.15WP (1×10 cfu/g) @ 2 kg/ha & $\begin{array}{l}6.24^{i} \\
(1.18)\end{array}$ & $\begin{array}{l}8.31^{\mathrm{e}} \\
(2.09)\end{array}$ & $\begin{array}{l}8.15^{\mathrm{e}} \\
(2.01)\end{array}$ & $\begin{array}{l}7.57^{9} \\
(1.74)\end{array}$ \\
\hline 10 & Castor cake@ 1 ton/ha & $\begin{array}{l}15.34^{c} \\
(7.00)\end{array}$ & $\begin{array}{l}15.93^{b} \\
(7.53)\end{array}$ & $\begin{array}{l}16.13^{b} \\
(7.72)\end{array}$ & $\begin{array}{l}15.80^{\mathrm{b}} \\
(7.41)\end{array}$ \\
\hline 11 & Castor cake@ 1 ton/ha + B. bassiana 1.15WP (1×10 cfu/g)@ 2 kg/ha & $\begin{array}{l}13.78^{\text {de }} \\
(5.67)\end{array}$ & $\begin{array}{l}12.56^{d} \\
(4.73)\end{array}$ & $\begin{array}{l}12.82^{d} \\
(4.92)\end{array}$ & $\begin{array}{l}13.06^{\text {de }} \\
(5.11)\end{array}$ \\
\hline 12 & Castor cake@ 1 ton/ha+M. anisopliae 1.15 WP (1 ×108 cfu/g)@ 2 kg/ha & $\begin{array}{l}6.94^{\mathrm{hi}} \\
(1.46)\end{array}$ & $\begin{array}{l}8.78^{\mathrm{e}} \\
(2.33)\end{array}$ & $\begin{array}{l}8.66^{\mathrm{e}} \\
(2.27)\end{array}$ & $\begin{array}{l}8.13^{9} \\
(2.00)\end{array}$ \\
\hline 13 & Neem cake@ 1 ton/ha & $\begin{array}{l}18.32^{\mathrm{a}} \\
(9.88)\end{array}$ & $\begin{array}{l}17.90^{\mathrm{a}} \\
(9.45)\end{array}$ & $\begin{array}{l}18.09^{\mathrm{a}} \\
(9.64)\end{array}$ & $\begin{array}{l}18.10^{\mathrm{a}} \\
(9.65)\end{array}$ \\
\hline \multirow[t]{7}{*}{ S.Em. \pm} & $\mathrm{T}$ & 0.395 & 0.435 & 0.427 & 0.510 \\
\hline & $\mathrm{P}$ & 0.219 & 0.241 & 0.237 & 0.310 \\
\hline & Y & - & - & - & 0.116 \\
\hline & $T \times P$ & 0.790 & 0.871 & 0.854 & 0.484 \\
\hline & $T \times Y$ & - & - & - & 0.419 \\
\hline & $P \times Y$ & - & - & - & 0.233 \\
\hline & $T \times P \times Y$ & - & - & - & 0.839 \\
\hline \multirow[t]{2}{*}{ C. D. at $5 \%$} & $\mathrm{~T}$ & 1.108 & 1.221 & 1.198 & 1.489 \\
\hline & $T \times P \times Y$ & & & & NS \\
\hline C.V. (\%) & & 11.36 & 11.40 & 11.10 & 11.30 \\
\hline
\end{tabular}

1. Figures in parentheses are retransformed values of arc sin transformation; DAG: Days after germination; 2 . Treatment means with the letter(s) in common are not significant by DNMRT at $5 \%$ level of significance; 3 . Significant parameters and their interactions: T, P, Y, T $\times P, T \times Y, P \times Y$

application of vermicompost @ 1 ton/ha + M. anisopliae 1.15 WP $\left(1 \times 10^{8} \mathrm{cfu} / \mathrm{g}\right) @ 2 \mathrm{~kg} / \mathrm{ha}$ was the most effective treatment and it was at par with castor cake @ 1 ton/ha + M. anisopliae 1.15 WP $\left(1 \times 10^{8} \mathrm{cfu} / \mathrm{g}\right) @ 2 \mathrm{~kg} /$ ha and neem cake@ 1 ton/ha + M. anisopliae 1.15 WP $\left(1 \times 10^{8} \mathrm{cfu} / \mathrm{g}\right) @ 2 \mathrm{~kg} / \mathrm{ha}$ (Table 6).

\section{Effect of entomopathogenic fungi enriched with organic amendments on number of white grub/meter row}

The perusal of data presented in Table 7 indicated that the plots treated with vermicompost @ 1 ton/ha $+M$. anisopliae 1.15 WP $\left(1 \times 10^{8} \mathrm{cfu} / \mathrm{g}\right) @ 2 \mathrm{~kg} / \mathrm{ha}$ registered the least $(0.19)$ white grub population per one meter row length during pooled results of the different 3 seasons and it was at par with par with castor cake @ 1 ton $/ \mathrm{ha}+$ M. anisopliae $1.15 \mathrm{WP}\left(1 \times 10^{8} \mathrm{cfu} / \mathrm{g}\right) @ 2 \mathrm{~kg} /$ ha and neem cake @ 1 ton/ha + M. anisopliae 1.15 WP 
Table 6 Effect of entomopathogenic fungi enriched with organic amendments against white grub in groundnut (pooled over seasons)

\begin{tabular}{|c|c|c|c|c|c|}
\hline \multirow[t]{2}{*}{ Tr. no } & \multirow[t]{2}{*}{ Treatments } & \multicolumn{3}{|c|}{ Cumulative dead plants (\%) } & \multirow{2}{*}{$\begin{array}{l}\text { Pooled } \\
\text { over } \\
\text { seasons }\end{array}$} \\
\hline & & 2018 & 2019 & 2020 & \\
\hline 1 & Neem cake@ 1 ton/ha & $\begin{array}{l}20.81^{\mathrm{ef}} \\
(12.64)\end{array}$ & $\begin{array}{l}32.10^{c} \\
(28.25)\end{array}$ & $\begin{array}{l}32.45^{c} \\
(28.79)\end{array}$ & $\begin{array}{l}28.44^{\mathrm{cd}} \\
(22.68)\end{array}$ \\
\hline 2 & Neem cake@ 1 ton/ha + Beauveria bassiana 1.15WP (1×108cu/g)@ 2 kg/ha & $\begin{array}{l}19.64^{f} \\
(11.31)\end{array}$ & $\begin{array}{l}27.02^{d} \\
(20.66)\end{array}$ & $\begin{array}{l}27.55^{d} \\
(21.39)\end{array}$ & $\begin{array}{l}24.73^{e} \\
(17.50)\end{array}$ \\
\hline 3 & Neem cake@ 1 ton/ha + Metarhizium anisopliae 1.15 WP $\left(1 \times 10^{8} \mathrm{cfu} / \mathrm{g}\right) @ 2$ kg/ha & $\begin{array}{l}16.17^{9} \\
(7.83)\end{array}$ & $\begin{array}{l}19.65^{e} \\
(11.40)\end{array}$ & $\begin{array}{l}19.22^{e} \\
(10.84)\end{array}$ & $\begin{array}{l}18.32^{f} \\
(9.88)\end{array}$ \\
\hline 4 & Poultry manure@ 1 ton/ha & $\begin{array}{l}30.60^{d} \\
(25.94)\end{array}$ & $\begin{array}{l}32.18^{c} \\
(28.38)\end{array}$ & $\begin{array}{l}32.58^{\mathrm{c}} \\
(28.99)\end{array}$ & $\begin{array}{l}31.78^{\mathrm{c}} \\
(27.74)\end{array}$ \\
\hline 5 & Poultry manure@1 ton/ha + B.bassiana 1.15 WP $\left(1 \times 10^{8} \mathrm{cfu} / \mathrm{g}\right) @ 2$ kg/ha & $\begin{array}{l}29.18^{d} \\
(23.79)\end{array}$ & $\begin{array}{l}31.90^{c} \\
(27.99)\end{array}$ & $\begin{array}{l}32.11^{c} \\
(28.26)\end{array}$ & $\begin{array}{l}31.04^{c} \\
(26.59)\end{array}$ \\
\hline 6 & Poultry manure@ 1 ton/ha+M. anisopliae 1.15 WP $\left(1 \times 10^{8} \mathrm{cfu} / \mathrm{g}\right) @ 2$ kg/ha & $\begin{array}{l}23.25^{\mathrm{e}} \\
(15.68)\end{array}$ & $\begin{array}{l}26.59^{d} \\
(20.04)\end{array}$ & $\begin{array}{l}27.00^{d} \\
(20.62)\end{array}$ & $\begin{array}{l}25.59^{\text {de }} \\
(18.66)\end{array}$ \\
\hline 7 & Vermicompost @ 1 ton/ha & $\begin{array}{l}38.09^{b} \\
(38.07)\end{array}$ & $\begin{array}{l}37.47^{b} \\
(37.02)\end{array}$ & $\begin{array}{l}37.40^{\mathrm{b}} \\
(36.89)\end{array}$ & $\begin{array}{l}37.64^{b} \\
(37.30)\end{array}$ \\
\hline 8 & Vermicompost @ 1 ton/ha +B.bassiana 1.15 WP $\left(1 \times 10^{8} \mathrm{cfu} / \mathrm{g}\right) @ 2$ kg/ha & $\begin{array}{l}33.96^{c} \\
(31.26)\end{array}$ & $\begin{array}{l}36.25^{b} \\
(35.01)\end{array}$ & $\begin{array}{l}36.65^{b} \\
(35.63)\end{array}$ & $\begin{array}{l}35.61^{b} \\
(33.90)\end{array}$ \\
\hline 9 & Vermicompost @ 1 ton/ha +M. anisopliae 1.15WP (1 × 10 cfu/g) @ 2 kg/ha & $\begin{array}{l}14.20^{9} \\
(6.05)\end{array}$ & $\begin{array}{l}17.79^{e} \\
(9.45)\end{array}$ & $\begin{array}{l}17.38^{\mathrm{e}} \\
(8.93)\end{array}$ & $\begin{array}{l}16.45^{f} \\
(8.02)\end{array}$ \\
\hline 10 & Castor cake@ 1 ton/ha & $\begin{array}{l}35.44^{b c} \\
(33.63)\end{array}$ & $\begin{array}{l}36.77^{b} \\
(35.96)\end{array}$ & $\begin{array}{l}37.29^{b} \\
(36.71)\end{array}$ & $\begin{array}{l}36.50^{b} \\
(35.38)\end{array}$ \\
\hline 11 & Castor cake@ 1 ton/ha+B. bassiana 1.15WP (1×10² cfu/g)@ 2 kg/ha & $\begin{array}{l}30.62^{d} \\
(25.96)\end{array}$ & $\begin{array}{l}27.52^{\mathrm{d}} \\
(21.39)\end{array}$ & $\begin{array}{l}28.12^{\mathrm{d}} \\
(22.22)\end{array}$ & $\begin{array}{l}28.75^{c d} \\
(23.14)\end{array}$ \\
\hline 12 & Castor cake@ 1 ton/ha+M. anisopliae 1.15WP (1×108 cfu/g)@ 2kg/ha & $\begin{array}{l}15.05^{\mathrm{g}} \\
(6.76)\end{array}$ & $\begin{array}{l}18.87^{e} \\
(10.51)\end{array}$ & $\begin{array}{l}18.53^{\mathrm{e}} \\
(10.10)\end{array}$ & $\begin{array}{l}17.49^{f} \\
(9.03)\end{array}$ \\
\hline 13 & Neem cake@ 1 ton/ha & $\begin{array}{l}42.58^{\mathrm{a}} \\
(45.80)\end{array}$ & $\begin{array}{l}41.98^{\mathrm{a}} \\
(44.78)\end{array}$ & $\begin{array}{l}42.47^{\mathrm{a}} \\
(45.60)\end{array}$ & $\begin{array}{l}42.32^{\mathrm{a}} \\
(45.33)\end{array}$ \\
\hline \multirow[t]{3}{*}{ S.Em. \pm} & $\mathrm{T}$ & 1.00 & 1.17 & 1.15 & 1.206 \\
\hline & Y & - & - & - & 0.308 \\
\hline & $T \times Y$ & - & - & - & 1.111 \\
\hline C. D. at $5 \%$ & $\mathrm{~T}$ & 2.91 & 3.42 & 3.37 & 3.52 \\
\hline C.V. (\%) & & 6.43 & 6.86 & 6.68 & 6.67 \\
\hline
\end{tabular}

1. Figures in parentheses are retransformed values of arc sin transformation; DAG: Days after germination; 2 . Treatment means with the letter(s) in common are not significant by DNMRT at $5 \%$ level of significance; 3 . Significant parameters and their interactions: $\mathrm{T}, \mathrm{T} \times \mathrm{Y}$

$\left(1 \times 10^{8} \mathrm{cfu} / \mathrm{g}\right) @ 2 \mathrm{~kg} / \mathrm{ha}$ with 0.26 and 0.31 white grub population per one meter row length, respectively.

\section{Effect of entomopathogenic fungi enriched with organic amendments on Pod yield ( $\mathrm{kg} / \mathrm{ha}$ )}

Looking to overall pooled results of seasons 2018, 2019 and 2020, it was clearly indicated that maximum pod yield was observed in the plot treated with soil application of vermicompost @ 1 ton/ha $+M$. anisopliae 1.15 WP $\left(1 \times 10^{8} \mathrm{cfu} / \mathrm{g}\right) @ 2 \mathrm{~kg} / \mathrm{ha}(1669 \mathrm{~kg} / \mathrm{ha})$ and it was remained at par with castor cake @ 1 ton/ha $+M$. anisopliae 1.15 WP $\left(1 \times 10^{8} \mathrm{cfu} / \mathrm{g}\right) @ 2 \mathrm{~kg} / \mathrm{ha}(1649 \mathrm{~kg} /$ ha) and neem cake @ 1 ton/ha + M. anisopliae 1.15 WP $\left(1 \times 10^{8} \mathrm{cfu} / \mathrm{g}\right) @ 2 \mathrm{~kg} / \mathrm{ha}(1633 \mathrm{~kg} / \mathrm{ha})$ (Table 8).

\section{Economics}

The data (Table 9) showed that maximum net realization and net gain was obtained in the treatment of vermicompost @ 1 ton/ha + M. anisopliae 1.15 WP $\left(1 \times 10^{8} \mathrm{cfu} / \mathrm{g}\right)$ @ $2 \mathrm{~kg} / \mathrm{ha}$, followed by castor cake @ 1 ton/ha $+M$. anisopliae 1.15 WP $\left(1 \times 10^{8} \mathrm{cfu} / \mathrm{g}\right) @ 2 \mathrm{~kg} / \mathrm{ha}$ and neem cake @ 1 ton/ha + M. anisopliae 1.15 WP $\left(1 \times 10^{8} \mathrm{cfu} / \mathrm{g}\right)$ @ $2 \mathrm{~kg} / \mathrm{h}$. The highest ICBR was recorded in the treatment of castor cake @ 1 ton/ha + M. anisopliae 1.15 WP $\left(1 \times 10^{8} \mathrm{cfu} / \mathrm{g}\right) @ 2 \mathrm{~kg} / \mathrm{ha}(1: 6.88)$.

\section{Discussion}

The majority of research on EPF has focused on developing them as inundative biological control agents for white grubs. The majority of commercially produced fungus is Beauveria or Metarhizium species, both of which are 
Table 7 Effect of entomopathogenic fungi enriched with organic amendments against white grub/1 m row in groundnut (pooled over seasons)

\begin{tabular}{|c|c|c|c|c|c|}
\hline \multirow[t]{2}{*}{ Tr. no } & \multirow[t]{2}{*}{ Treatments } & \multicolumn{3}{|c|}{ White grub/1 m row } & \multirow{2}{*}{$\begin{array}{l}\text { Pooled } \\
\text { over } \\
\text { seasons }\end{array}$} \\
\hline & & 2018 & 2019 & 2020 & \\
\hline 1 & Neem cake@ 1 ton/ha & $\begin{array}{l}0.93^{e f} \\
(0.36)\end{array}$ & $\begin{array}{l}1.16^{\mathrm{d}} \\
(0.86)\end{array}$ & $\begin{array}{l}1.13^{c} \\
(0.77)\end{array}$ & $\begin{array}{l}1.07^{\mathrm{de}} \\
(0.64)\end{array}$ \\
\hline 2 & Neem cake@ 1 ton/ha + Beauveria bassiana 1.15WP (1×108cu/g)@ 2kg/ha & $\begin{array}{l}0.91^{e f} \\
(0.33)\end{array}$ & $\begin{array}{l}1.05^{\mathrm{de}} \\
(0.60)\end{array}$ & $\begin{array}{l}1.10^{c} \\
(0.71)\end{array}$ & $\begin{array}{l}1.01^{\mathrm{e}} \\
(0.52)\end{array}$ \\
\hline 3 & Neem cake@ 1 ton/ha + Metarhizium anisopliae 1.15 WP (1 × 108 cfu/g) @ 2 kg/ha & $\begin{array}{l}0.89^{\mathrm{ef}} \\
(0.29)\end{array}$ & $\begin{array}{l}0.91^{\text {ef }} \\
(0.33)\end{array}$ & $\begin{array}{l}0.90^{d} \\
(0.32)\end{array}$ & $\begin{array}{l}0.90^{f} \\
(0.31)\end{array}$ \\
\hline 4 & Poultry manure @ 1 ton/ha & $\begin{array}{l}1.20^{\mathrm{bcd}} \\
(0.94)\end{array}$ & $\begin{array}{l}1.20^{\mathrm{cd}} \\
(0.94)\end{array}$ & $\begin{array}{l}1.17^{c} \\
(0.87)\end{array}$ & $\begin{array}{l}1.18^{c} \\
(0.89)\end{array}$ \\
\hline 5 & Poultry manure@ 1 ton/ha + B. bassiana 1.15 WP $\left(1 \times 10^{8} \mathrm{cfu} / \mathrm{g}\right) @ 2$ kg/ha & $\begin{array}{l}1.16^{\mathrm{cd}} \\
(0.85)\end{array}$ & $\begin{array}{l}1.16^{\mathrm{d}} \\
(0.86)\end{array}$ & $\begin{array}{l}1.12^{c} \\
(0.76)\end{array}$ & $\begin{array}{l}1.14^{\mathrm{cd}} \\
(0.80)\end{array}$ \\
\hline 6 & Poultry manure @ 1 ton/ha + M. anisopliae 1.15WP (1×10 cfu/g)@2kg/ha & $\begin{array}{l}1.07^{\mathrm{de}} \\
(0.64)\end{array}$ & $\begin{array}{l}1.05^{\mathrm{de}} \\
(0.60)\end{array}$ & $\begin{array}{l}1.09^{c} \\
(0.68)\end{array}$ & $\begin{array}{l}1.06^{\mathrm{de}} \\
(0.62)\end{array}$ \\
\hline 7 & Vermicompost @ 1 ton/ha & $\begin{array}{l}1.39^{b} \\
(1.43)\end{array}$ & $\begin{array}{l}1.39^{\mathrm{b}} \\
(1.45)\end{array}$ & $\begin{array}{l}1.42^{\mathrm{b}} \\
(1.50)\end{array}$ & $\begin{array}{l}1.39^{b} \\
(1.43)\end{array}$ \\
\hline 8 & Vermicompost@1ton/ha+B.bassiana 1.15WP (1×10 cfu/g)@ 2 kg/ha & $\begin{array}{l}1.36^{\mathrm{bc}} \\
(1.35)\end{array}$ & $\begin{array}{l}1.35^{\mathrm{bc}} \\
(1.33)\end{array}$ & $\begin{array}{l}1.38^{\mathrm{b}} \\
(1.40)\end{array}$ & $\begin{array}{l}1.36^{b} \\
(1.35)\end{array}$ \\
\hline 9 & Vermicompost@ 1 ton/ha+M. anisopliae 1.15WP (1×108fu/g)@ 2 kg/ha & $\begin{array}{l}0.84^{f} \\
(0.21)\end{array}$ & $\begin{array}{l}0.85^{f} \\
(0.22)\end{array}$ & $\begin{array}{l}0.82^{d} \\
(0.17)\end{array}$ & $\begin{array}{l}0.83^{f} \\
(0.19)\end{array}$ \\
\hline 10 & Castor cake@ 1 ton/ha & $\begin{array}{l}1.35^{\mathrm{bc}} \\
(1.32)\end{array}$ & $\begin{array}{l}1.35^{b c} \\
(1.36)\end{array}$ & $\begin{array}{l}1.39^{\mathrm{b}} \\
(1.43)\end{array}$ & $\begin{array}{l}1.36^{b} \\
(1.35)\end{array}$ \\
\hline 11 & Castor cake@ 1 ton/ha + B.bassiana 1.15WP (1×10cfu/g)@2kg/ha & $\begin{array}{l}1.29^{b c d} \\
(1.16)\end{array}$ & $\begin{array}{l}1.06^{\mathrm{de}} \\
(0.63)\end{array}$ & $\begin{array}{l}1.12^{c} \\
(0.75)\end{array}$ & $\begin{array}{l}1.15^{c d} \\
(0.82)\end{array}$ \\
\hline 12 & 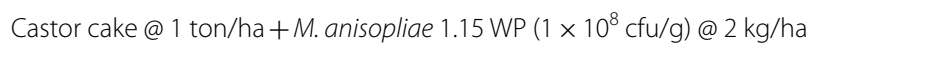 & $\begin{array}{l}0.86^{\mathrm{ef}} \\
(0.24)\end{array}$ & $\begin{array}{l}0.89^{e f} \\
(0.30)\end{array}$ & $\begin{array}{l}0.86^{d} \\
(0.24)\end{array}$ & $\begin{array}{l}0.87^{\mathrm{f}} \\
(0.26)\end{array}$ \\
\hline 13 & Neem cake@ @ ton/ha & $\begin{array}{l}1.70^{\mathrm{a}} \\
(2.39)\end{array}$ & $\begin{array}{l}1.63^{\mathrm{a}} \\
(2.18)\end{array}$ & $\begin{array}{l}1.66^{\mathrm{a}} \\
(2.24)\end{array}$ & $\begin{array}{l}1.66^{\mathrm{a}} \\
(2.26)\end{array}$ \\
\hline \multirow[t]{3}{*}{ S.Em. \pm} & T & 0.07 & 0.06 & 0.06 & 0.035 \\
\hline & Y & - & - & - & 0.017 \\
\hline & $T \times Y$ & - & - & - & 0.061 \\
\hline \multirow[t]{2}{*}{ C.D. at $5 \%$} & $T$ & 0.19 & 0.18 & 0.16 & 0.10 \\
\hline & $T \times Y$ & - & - & - & NS \\
\hline C.V. (\%) & & 9.86 & 9.36 & 8.23 & 9.17 \\
\hline
\end{tabular}

1. Figures in parentheses are retransformed values of $\sqrt{x+0.5}$ transformation; DAG: Days after germination; 2 . Treatment means with the letter(s) in common are not significant by DNMRT at $5 \%$ level of significance

very easy to mass-produce. Production criteria include low cost, long-term stability, and, most significantly, consistent efficacy in the field. The development of a suitable formulation is mandatory in order to enhance spore application and successful utilization in soil (Chandel et al. 2018). According to Arora et al. (2000), entomopathogens control white grubs by invasive characteristics, poisons, enzymes, and other compounds. EPF attracted greater attention than other families of microbial organisms with the potential to be used in white grub management.

The present study clearly indicated that minimum plant mortality due to white grub was recorded in the plot treated with soil application of vermicompost @ 1 ton/ha + M. anisopliae 1.15 WP $\left(1 \times 10^{8} \mathrm{cfu} / \mathrm{g}\right) @ 2 \mathrm{~kg} /$ ha and it was at part with castor cake@ 1 ton/ha $+M$. anisopliae 1.15 WP $\left(1 \times 10^{8} \mathrm{cfu} / \mathrm{g}\right) @ 2 \mathrm{~kg} / \mathrm{ha}$ and neem cake @ 1 ton/ha + M. anisopliae 1.15 WP $\left(1 \times 10^{8} \mathrm{cfu} / \mathrm{g}\right)$ @ $2 \mathrm{~kg} / \mathrm{ha}$, followed by B. bassiana enriched with organic amendments. The current findings are line with the observations of other researchers, such as Mohi-Ud-Din et al. (2006), who indicated that M. Anisopliae, B. bassiana and $B$. brongniartii were very efficient against white grubs in Kashmir at $1 \times 10^{8}$ spores/ml. After 20-24 days of therapy, these cultures resulted in overall mortality of $100 \%$. Kulye and Pokharkar (2009) studied the efficacy of $M$. anisopliae and B. bassiana against $H$. consanguinea infesting potato. Use of M. anisopliae @ $2 \times 10^{12}$ 
Table 8 Effect of entomopathogenic fungi enriched with organic amendments against white grub on yield of groundnut (Pooled over seasons)

\begin{tabular}{|c|c|c|c|c|c|}
\hline \multirow[t]{2}{*}{ Tr. no } & \multirow[t]{2}{*}{ Treatments } & \multicolumn{3}{|c|}{ Yield (kg/ha) } & \multirow{2}{*}{$\begin{array}{l}\text { Pooled } \\
\text { over } \\
\text { seasons }\end{array}$} \\
\hline & & 2018 & 2019 & 2020 & \\
\hline 1 & Neem cake@ 1 ton/ha & $1426^{\mathrm{abc}}$ & $1358^{b}$ & $1367^{b}$ & $1384^{b}$ \\
\hline 2 & Neem cake@ 1 ton/ha + Beauveria bassiana 1.15WP $\left(1 \times 10^{8} \mathrm{cfu} / \mathrm{g}\right) @ 2 \mathrm{~kg} / \mathrm{ha}$ & $1440^{\mathrm{ab}}$ & $1400^{b}$ & $1422^{b}$ & $1421^{b}$ \\
\hline 3 & Neem cake@ 1 ton/ha + Metarhizium anisopliae 1.15WP $\left(1 \times 10^{8} \mathrm{cfu} / \mathrm{g}\right) @ 2 \mathrm{~kg} / \mathrm{ha}$ & $1585^{\mathrm{a}}$ & $1650^{\mathrm{a}}$ & $1664^{\mathrm{a}}$ & $1633^{\mathrm{a}}$ \\
\hline 4 & Poultry manure @ 1 ton/ha & $1197^{\mathrm{bcd}}$ & $1207^{\mathrm{bcd}}$ & $1237^{b c}$ & $1215^{c}$ \\
\hline 5 & Poultry manure@ 1 ton/ha + B. bassiana 1.15WP $\left(1 \times 10^{8} \mathrm{cfu} / \mathrm{g}\right) @ 2 \mathrm{~kg} / \mathrm{ha}$ & $1225^{\mathrm{bcd}}$ & $1242^{\mathrm{bc}}$ & $1260^{\mathrm{bc}}$ & $1242^{c}$ \\
\hline 6 & 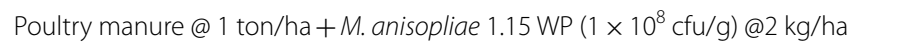 & $1396^{\mathrm{abc}}$ & $1417^{b}$ & $1431^{b}$ & $1415^{b}$ \\
\hline 7 & Vermicompost@ 1 ton/ha & $988^{\text {de }}$ & $997^{\text {de }}$ & $1049^{\mathrm{cd}}$ & $1011^{d}$ \\
\hline 8 & 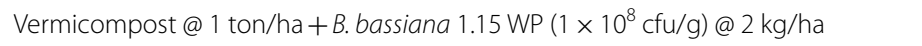 & $1049^{\text {de }}$ & $1083^{\text {cde }}$ & $1105^{\mathrm{cd}}$ & $1079^{d}$ \\
\hline 9 & Vermicompost@ 1 ton/ha + M. anisopliae 1.15WP $\left(1 \times 10^{8} \mathrm{cfu} / \mathrm{g}\right) @ 2$ kg/ha & $1628^{\mathrm{a}}$ & $1675^{\mathrm{a}}$ & $1704^{\mathrm{a}}$ & $1669^{\mathrm{a}}$ \\
\hline 10 & Castor cake@ 1 ton/ha & $853^{\mathrm{ef}}$ & $867^{\mathrm{ef}}$ & $927^{\text {de }}$ & $882^{e}$ \\
\hline 11 & 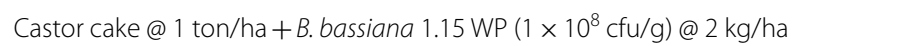 & $1174^{\mathrm{cd}}$ & $1191^{\mathrm{bcd}}$ & $1219^{\mathrm{bc}}$ & $1195^{c}$ \\
\hline 12 & Castor cake@ 1 ton/ha+M. anisopliae 1.15WP (1×108 cfu/g)@ 2 kg/ha & $1599^{\mathrm{a}}$ & $1660^{\mathrm{a}}$ & $1688^{\mathrm{a}}$ & $1649^{\mathrm{a}}$ \\
\hline 13 & Neem cake@ 1 ton/ha & $720^{f}$ & $758^{f}$ & $801^{\mathrm{e}}$ & $760^{f}$ \\
\hline \multirow[t]{3}{*}{ S.Em. \pm} & T & 78.47 & 75.41 & 70.80 & 38.102 \\
\hline & Y & - & - & - & 20.790 \\
\hline & $T \times Y$ & - & - & - & 74.958 \\
\hline \multirow[t]{2}{*}{ C. D. at $5 \%$} & $\mathrm{~T}$ & 229.02 & 220.10 & 206.66 & 106.96 \\
\hline & $T \times Y$ & - & - & - & NS \\
\hline C.V. (\%) & & 10.85 & 10.29 & 9.45 & 10.20 \\
\hline
\end{tabular}

1. DAG: Days after germination; Treatment means with the letter(s) in common are not significant by DNMRT at $5 \%$ level of significance

conidia/ha showed average efficacy of $46.74 \%$, with $44.44 \%$ mycosis of grubs. Similarly, Yadava and Sharma (1995) reported that M. anisopliae and B. bassiana are pathogenic to white grubs and effective in suppressing their population under field conditions. Application of $M$. anisopliae in sugarcane @ $4 \times 10^{9}$ conidia/ha registered $92 \%$ reduction in grub population of Holotrichia serrata (Fab) in Tamil Nadu on $60^{\text {th }}$ DAT (Manisegaran et al. 2011). Among the treatments imposed at the time of sowing, M. anisopliae @ $5.0 \times 10^{13}$ spores/ha mixed with FYM was found effective, followed by $B$. bassiana @ $5.0 \times 10^{13}$ spores/ha applied in FYM enriched field and registered 93.60 and $88.09 \%$ decrease in white grub damage and 77.22 and $74.08 \%$ decrease in white grub population (Visalakshi et al. 2015). Avasthy (1967) reported good control of white grubs in India by $M$. anisopliae. Contrary to this, Chandel and Mehta (2005) tested a Jaipur culture of B. bassiana and M. anisopliae @ $5 \times 10^{13}$ conidia/ha against potato white grubs (Brahmina coriacea Hope) in Shimla hills, but neither of these fungi were found to be effective. Similarly, Chandel et al. (2005) did not find satisfactory control of $B$. coriacea grubs in potato in Shimla hills with the application of $B$. bassiana and $M$. anisopliae dusts.

In the present study, EPF in combination with organic amendments, such as vermicompost, neem cake and castor cake gave effective results against damage caused by the white grub. Literature scanning revealed that information pertaining to the evaluation of mixture of EPF and organic amendments against white grub is scanty. Hence, this study will be established as a future reference for further investigations on these combined products against the white grub and other soil-dwelling insect-pests. The study indicated that all the test products had variable insecticidal properties and may be used for the pest control at the time of sowing of the crop. According to the results of present study, the maximum net realization and net gain was obtained in the soil treatment of vermicompost @ 1 ton/ha $+M$. anisopliae 1.15 WP $\left(1 \times 10^{8} \mathrm{cfu} / \mathrm{g}\right) @ 2 \mathrm{~kg} / \mathrm{ha}$ and highest ICBR was recorded in the treatment of castor cake @ 1 ton/ ha $+M$. anisopliae 1.15 WP $\left(1 \times 10^{8} \mathrm{cfu} / \mathrm{g}\right) @ 2 \mathrm{~kg} / \mathrm{ha}(1$ : 6.88). Results of the present study are supported by Manisegaran et al. (2011) who observed higher incremental benefit-cost ratio (IBCR) with M. anisopliae (7.58) as compared to other chemical treatments.

\section{Conclusions}

White grubs are the most damaging soil pests inflicting damage to a groundnut crop, resulting in economic yield losses in India's commercial groundnut production. The ability of two EPF viz., $M$. anisopliae and $B$. 
Table 9 Economics of entomopathogenic fungi enriched with organic amendments evaluated against white grub in groundnut

\begin{tabular}{|c|c|c|c|c|c|c|c|c|c|}
\hline Tr. no & Treatments & $\begin{array}{l}\text { Cost of material } \\
\text { (Rs. /ha) }\end{array}$ & $\begin{array}{l}\text { Labor } \\
\text { charges } \\
\text { (Rs. /ha) }\end{array}$ & $\begin{array}{l}\text { Total cost of } \\
\text { treatment (Rs. } \\
\text { /ha) }\end{array}$ & $\begin{array}{l}\text { Yield } \\
\text { (Kg/ha) }\end{array}$ & $\begin{array}{l}\text { Gross } \\
\text { realization } \\
\text { (Rs. /ha) }\end{array}$ & $\begin{array}{l}\text { Net } \\
\text { realization } \\
\text { over control } \\
\text { (Rs. /ha) }\end{array}$ & $\begin{array}{l}\text { Net gain } \\
\text { (Rs. /ha) }\end{array}$ & PCBR \\
\hline 1 & Neem cake @ 1 ton/ha & 12,000 & 648 & 12,648 & 1384 & 73,006 & 32,916 & 20,268 & $1: 1.60$ \\
\hline 2 & $\begin{array}{l}\text { Neem cake @ } 1 \text { ton/ha }+ \text { Beau- } \\
\text { veriabassiana } 1.15 \text { WP } \\
\left(1 \times 10^{8} \mathrm{cfu} / \mathrm{g}\right) @ 2 \mathrm{~kg} / \mathrm{ha}\end{array}$ & 12,300 & 648 & 12,948 & 1421 & 74,958 & 34,868 & 21,920 & 1: 1.69 \\
\hline 3 & $\begin{array}{l}\text { Neem cake @ } 1 \text { ton/ } \\
\text { ha+ Metarhizium anisopliae } \\
1.15 \text { WP }\left(1 \times 10^{8} \mathrm{cfu} / \mathrm{g}\right) @ 2 \mathrm{~kg} / \\
\text { ha }\end{array}$ & 12,300 & 648 & 12,948 & 1633 & 86,141 & 46,051 & 33,103 & $1: 2.56$ \\
\hline 4 & Poultrymanure@1 ton/ha & 5000 & 648 & 5648 & 1214 & 64,039 & 23,949 & 18,301 & $1: 3.24$ \\
\hline 5 & $\begin{array}{l}\text { Poultry manure @ } 1 \text { ton/ } \\
\text { ha+B.bassiana } 1.15 \mathrm{WP} \\
\left(1 \times 10^{8} \mathrm{cfu} / \mathrm{g}\right) @ 2 \mathrm{~kg} / \mathrm{ha}\end{array}$ & 5300 & 648 & 5948 & 1242 & 65,516 & 25,426 & 19,478 & $1: 3.27$ \\
\hline 6 & $\begin{array}{l}\text { Poultry manure @ } 1 \text { ton/ } \\
\text { ha+M.anisopliae 1.15WP } \\
\left(1 \times 10^{8} \mathrm{cfu} / \mathrm{g}\right) @ 2 \mathrm{~kg} / \mathrm{ha}\end{array}$ & 5300 & 648 & 5948 & 1415 & 74,641 & 34,551 & 28,603 & $1: 4.81$ \\
\hline 7 & Vermicompost @ 1 ton/ha & 6000 & 648 & 6648 & 1011 & 53,330 & 13,240 & 6592 & 1:0.99 \\
\hline 8 & $\begin{array}{l}\text { Vermicompost @ } 1 \text { ton/ } \\
\text { ha+B.bassiana } 1.15 \text { WP } \\
\left(1 \times 10^{8} \mathrm{cfu} / \mathrm{g}\right) @ 2 \mathrm{~kg} / \mathrm{ha}\end{array}$ & 6300 & 648 & 6948 & 1079 & 56,917 & 16,827 & 9879 & $1: 1.42$ \\
\hline 9 & $\begin{array}{l}\text { Vermicompost @ } 1 \text { ton/ } \\
\text { ha+M.anisopliae 1.15WP } \\
\left(1 \times 10^{8} \mathrm{cfu} / \mathrm{g}\right) @ 2 \mathrm{~kg} / \mathrm{ha}\end{array}$ & 6300 & 648 & 6948 & 1669 & 88,040 & 47,950 & 41,002 & $1: 5.90$ \\
\hline 10 & Castor cake@ 1 ton/ha & 5000 & 648 & 5648 & 882 & 46,526 & 6436 & 788 & $1: 0.14$ \\
\hline 11 & $\begin{array}{l}\text { Castor cake @ } 1 \text { ton/ } \\
\text { ha+B.bassiana } 1.15 \text { WP } \\
\left(1 \times 10^{8} \mathrm{cfu} / \mathrm{g}\right) @ 2 \mathrm{~kg} / \mathrm{ha}\end{array}$ & 5300 & 648 & 5948 & 1195 & 63,036 & 22,946 & 16,998 & $1: 2.86$ \\
\hline 12 & $\begin{array}{l}\text { Castor cake @ } 1 \text { ton/ } \\
\text { ha+M.anisopliae 1.15 WP } \\
\left(1 \times 10^{8} \mathrm{cfu} / \mathrm{g}\right) @ 2 \mathrm{~kg} / \mathrm{ha}\end{array}$ & 5300 & 648 & 5948 & 1649 & 86,985 & 46,895 & 40,947 & 1: 6.88 \\
\hline 13 & Neem cake @ 1 ton/ha & - & - & - & 760 & 40,090 & - & - & - \\
\hline
\end{tabular}

Neem cake: 12 Rs/kg; Beauveria bassiana: 150 Rs/kg

Poultry manure:5 Rs/kg; Metarhizium anisopliae: $150 \mathrm{Rs} / \mathrm{kg}$

Vermicompost: 6 Rs/kg; Groundnut: 52.75 Rs/kg

Castor cake: 5 Rs/kg; Labor cost: 324 Rs/day

Labor required: $2 /$ day/ha

bassiana, supplemented with various organic amendments, to regulate the establishment of white grub populations in groundnut crops was investigated in this study. Based on the minimum plant mortality due to white grub, minimum white grub per one meter row length, highest pod yield, net realization and net gain, the treatment with soil application of vermicompost @ 1 ton/ha + M. anisopliae 1.15 WP $\left(1 \times 10^{8} \mathrm{cfu} / \mathrm{g}\right) @$ $2 \mathrm{~kg} / \mathrm{ha}$, castor cake @ 1 ton/ha + M. anisopliae 1.15 WP $\left(1 \times 10^{8} \mathrm{cfu} / \mathrm{g}\right) @ 2 \mathrm{~kg} / \mathrm{ha}$ and neem cake @ $1 \mathrm{ton} /$ ha + M. anisopliae 1.15 WP $\left(1 \times 10^{8} \mathrm{cfu} / \mathrm{g}\right) @ 2 \mathrm{~kg} / \mathrm{ha}$ were found the most effective treatments for the management of white grub in groundnut. Results indicated that $M$. anisopliae enriched with various organic amendments, such as vermicompost, castor cake, and neem cake are better alternatives for the management of white grubs in endemic areas at the time of sowing of the crop.

\section{Abbreviations}

ha: Hectare; g: Gram; kg: Kilogram; WP: Wettable powder; cfu: Colony-forming unit; mm: Millimeter; m: Meter; IPM: Integrated pest management; SDAU: Sardarkrushinagar Dantiwada agricultural university; ft: Feet; ASL: Above sea level; GG-2: Gujarat groundnut-2; cm: Centimeter; ICBR: Incremental cost-benefit ratio; FYM: Farm yard manure; DAG: Day after germination.

\section{Acknowledgements}

Authors are thankful to the Head, Department of Entomology, S. D. A. U., Sardarkrushinagar, Gujarat, India, for the financial support rendered in the form of a Research Project. 


\section{Authors' contributions}

PSP performed field trials, analyzed and interpreted the data of the work and prepared the original manuscript; SD conducted field trials, analyzed the data, reviewed and edited the writing. PHR assisted in conduction of field trials, analyzed the data. MJJ assisted in field trials, analyzed the data, reviewed and edited the manuscripts. The authors read and approved the final manuscript.

\section{Funding}

This work was supported by the Head, Department of Entomology, S. D. A. U., Sardarkrushinagar, Gujarat, India.

\section{Availability of data and materials}

All data generated and analyzed for the current study are presented in this manuscript, and the corresponding authors have no objection to the availability of data and materials.

\section{Declarations}

Ethics approval and consent to participate

Not applicable.

\section{Consent for publication}

The authors agreed to publish this paper. The data have not been published partially or completely in any other journal.

\section{Competing interests}

The authors declare that they have no competing interests.

Received: 20 October 2021 Accepted: 6 January 2022

Published online: 19 January 2022

\section{References}

Arora R, Battu GS, Ramakrishnan N (2000) Microbial pesticides: current status and future outlook. In: Dhaliwal GS, Singh B (eds) Pesticides and environment. Commonwealth Publishers, New Delhi

Avasthy PN (1967) Sugarcane pests in India and their control (a review). PANS 13:111-117

Chandel RS, Chandla VK, Dhiman KR (2005) Vulnerability of potato white grubs to entomogenous fungi and nematodes. Potato J 32(3-4):193-194

Chandel RS, Pathania M, Verma KS, Bhattacharyya B, Vashisth S, Kumar V (2015) The ecology and control of potato white grubs of India. Potato Res 58:147-164

Chandel RS, Soni S, Vashisth S, Pathania M, Mehta PK, Rana A, Bhatnagar A, Agrawal VK (2018) The potential of entomopathogens in biological control of white grubs. Int J Pest Manag. https://doi.org/10.1080/09670 874.2018 .1524183

Chandel YS, Mehta PK (2005) Efficacy of some insecticides and entomopathogenic fungi against white grub complex infesting potato in H.P. Indian J Ecol 32:195-199

Deepthi KC (2014) Effect of potential biocontrol agents against Sclerotium rolfsii causing stem rot of groundnut. Int J Life Sc Bt Pharm Res 2(2):58-65

Gomez KA, Gomez AA (1984) Statistical procedures for agricultural research, 2nd edn. Wiley, New York

Janila P, Nigam SN, Pandey MK, Nagesh P, Varshney RK (2013) Groundnut improvement: use of genetic and genomic tools. Front Plant Sci 4:23. https://doi.org/10.3389/fpls.2013.00023

Kulye MS, Pokharkar DS (2009) Evaluation of two species of entomopathogenic fungi against white grub, Holotrichia consanguinea (Blan.) infesting potato in Maharashtra. India J Biol Cont 23(1):1-4

Kumawat KC (2001) Evaluation of some insecticides against field termites in wheat. Ann PI Protec Sci 9:51-53

Manisegaran S, Lakshmi SM, Srimohanpriya V (2011) Field Evaluation of Metarhizium anisopliae (Metschnikoff) Sorokin against Holotrichia serrata (Blanch) in sugarcane. J Bio Pesticides 4(2):190-193

Mohi-Ud-Din S, Zaki FA, Munshi NA, Jan A, Sultan P (2006) Evaluation of some entomopathogenic fungal isolates from Kashmir for the biocontrol of white grubs infesting turf grass in golf course. J Biol Cont 20(1):45-50. https://doi.org/10.18311/jbc/2006/3941
Singh TVK, Singh KM, Singh RN (1990) Groundnut pest complex: III. Incidence of insect pests in relation to agro-climatic condition as determined by graphical super imposition technique. Ind J Ent 52(4):686-692

Veeresh GK (1988) White grubs. In: Veeresh GK, Rajgopal D (eds) Applied soil biology and ecology, 2nd edn. IBH, New Delhi

Visalakshi M, Bhavani B, Govinda RS (2015) Field evaluation of entomopathogenic fungi against white grub, Holotrichia consanguinea Blanchard in sugarcane. J Biol Cont 29(2):218-224

Yadava CPS, Sharma GK (1995) Indian white grubs and their management. Technical Bull, Indian Council of Agricultural Research, New Delhi, No. 2 p 26.

\section{Publisher's Note}

Springer Nature remains neutral with regard to jurisdictional claims in published maps and institutional affiliations.

\section{Submit your manuscript to a SpringerOpen ${ }^{\circ}$ journal and benefit from:}

- Convenient online submission

- Rigorous peer review

- Open access: articles freely available online

- High visibility within the field

- Retaining the copyright to your article

Submit your next manuscript at $\boldsymbol{\nabla}$ springeropen.com 